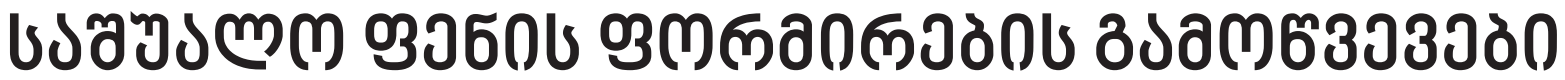

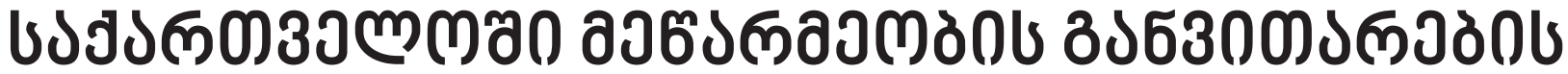 उलБООปЈ000
}

\section{১а0๓}

\author{
एलJ力m
}

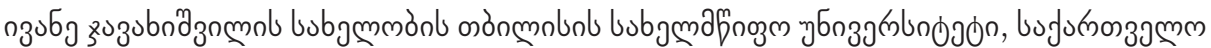

maglakelidze.amirani@gmail.com

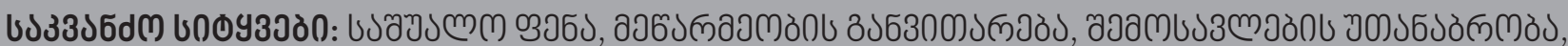

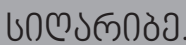

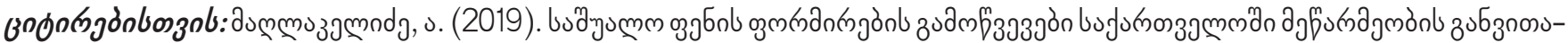

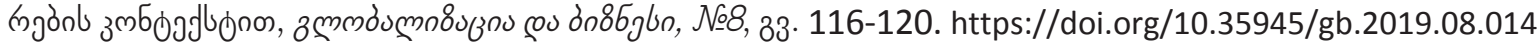

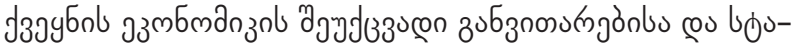

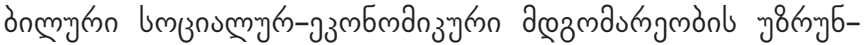

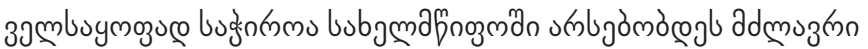

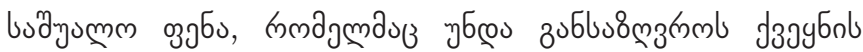

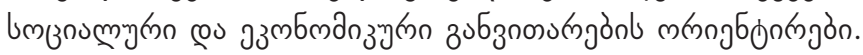

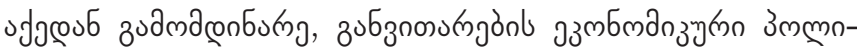

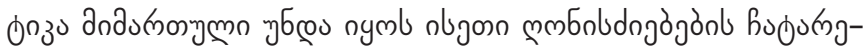

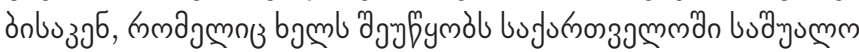

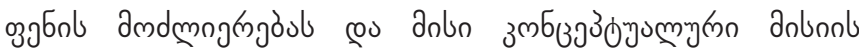
zubbmingngmgäl.

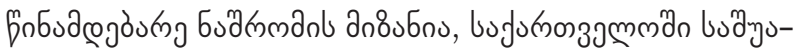

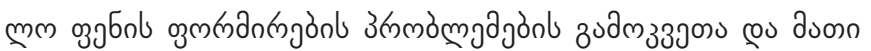

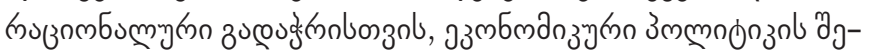

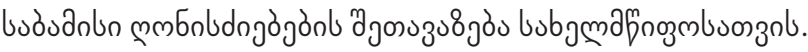

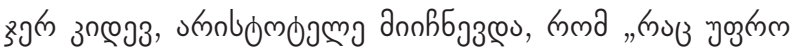

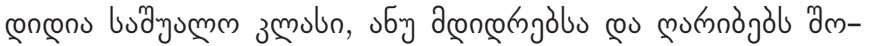
mol s s.

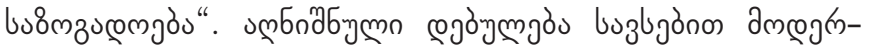

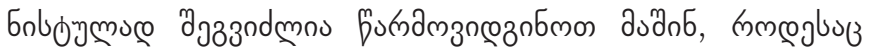

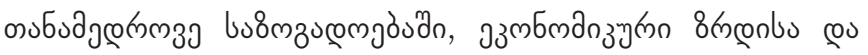

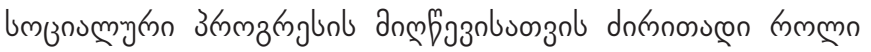

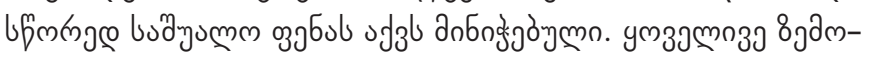

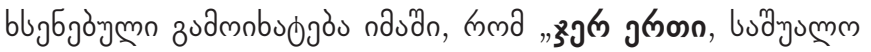

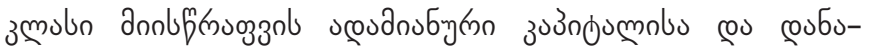

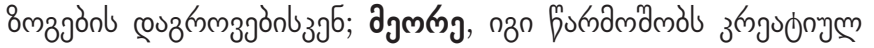

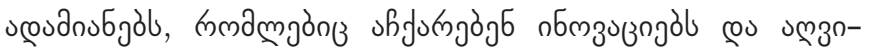

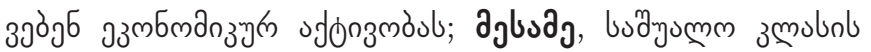

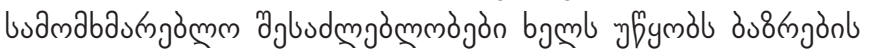

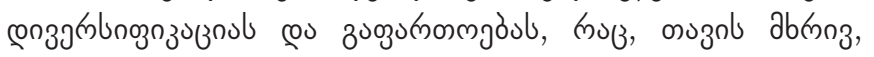

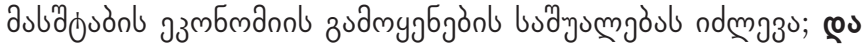

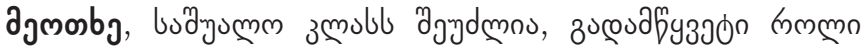

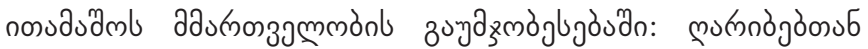

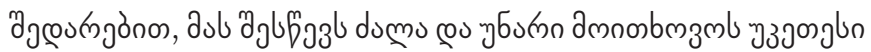

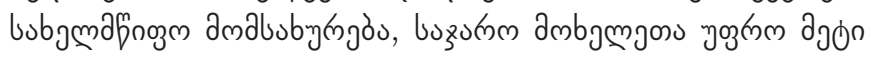

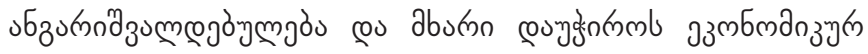
8кnূo

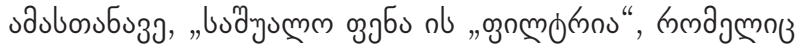

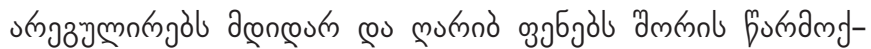

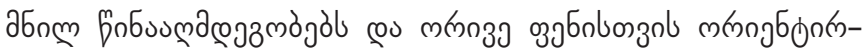

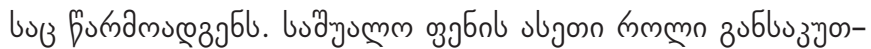

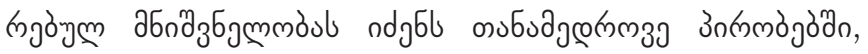

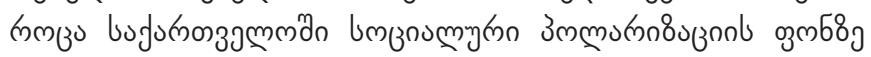

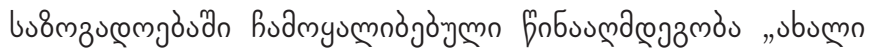

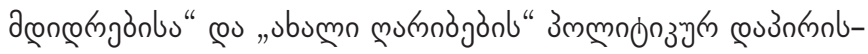

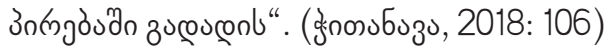

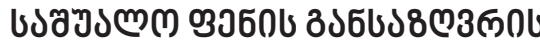

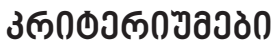

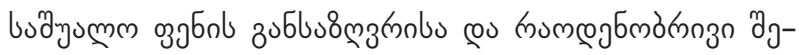

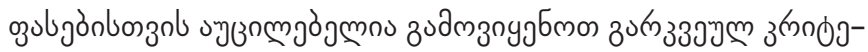

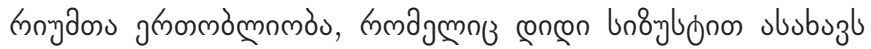

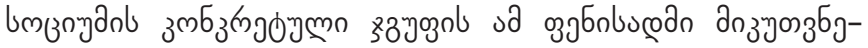

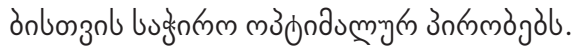

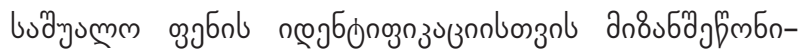

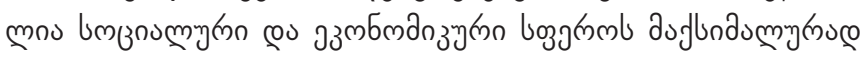

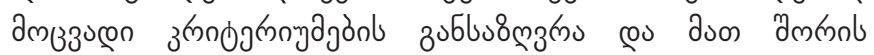




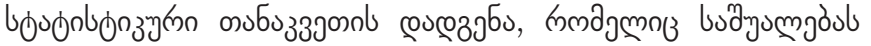

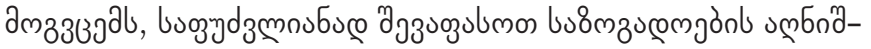

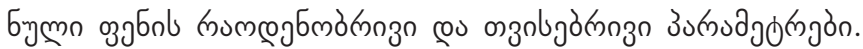

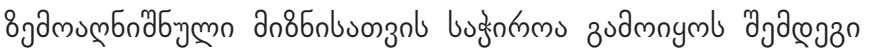

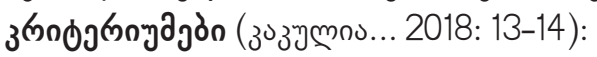

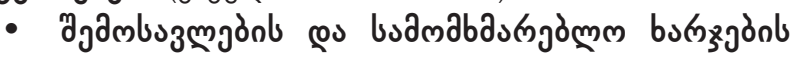

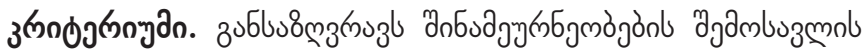

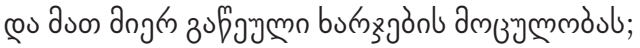

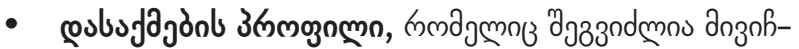

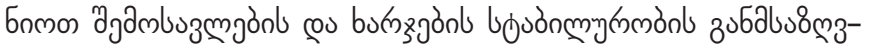

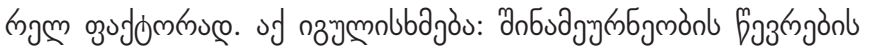

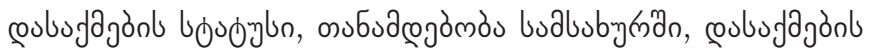

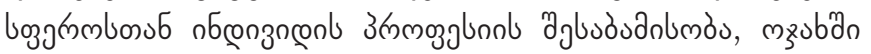

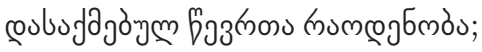

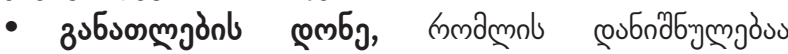

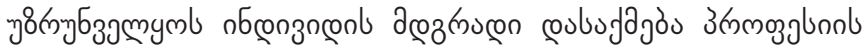

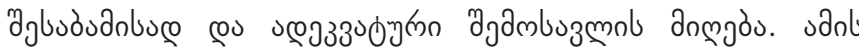

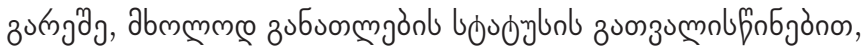

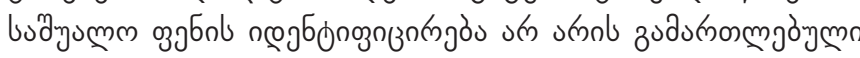

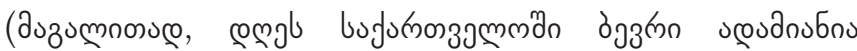

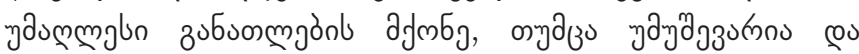

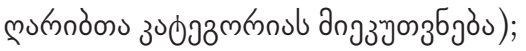

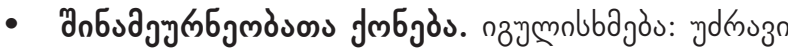

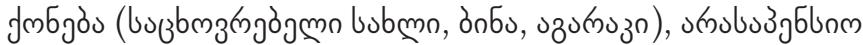

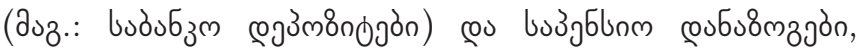

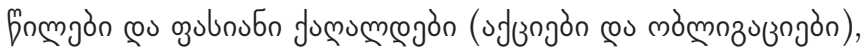

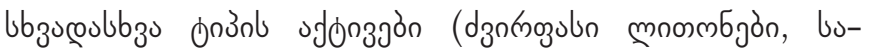

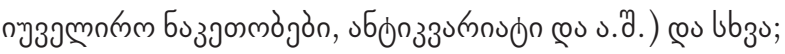

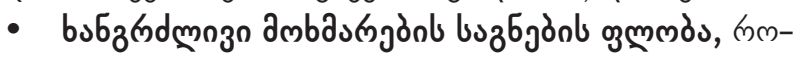

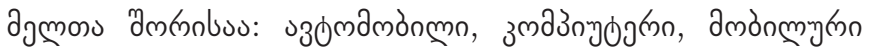

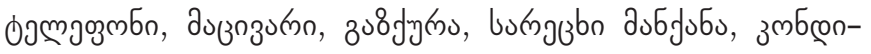

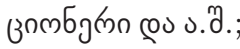

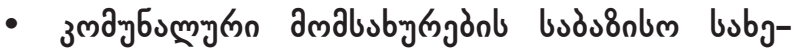

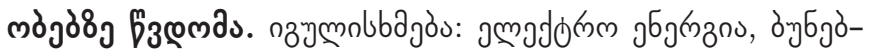

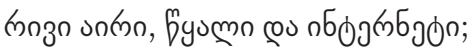

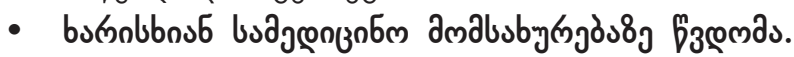

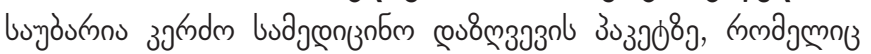

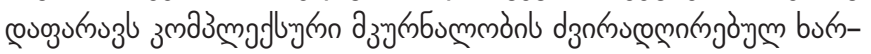

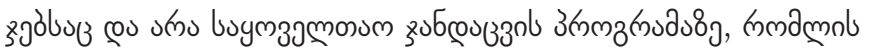

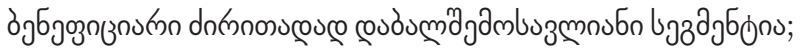

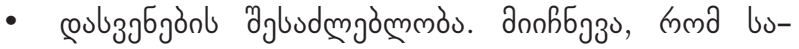

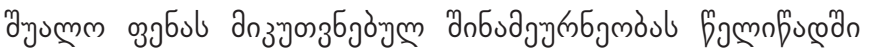

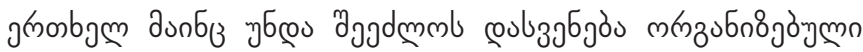

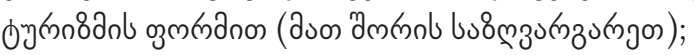

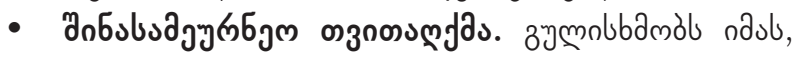

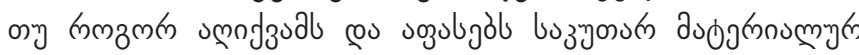

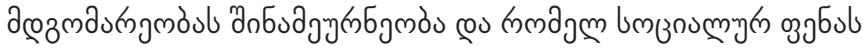

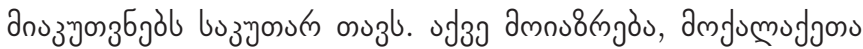

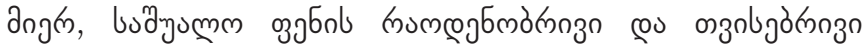
aububnuogòmgònb unjas.

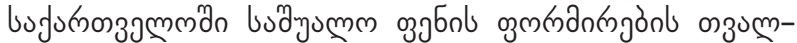

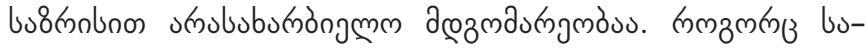

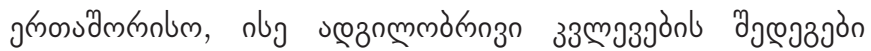

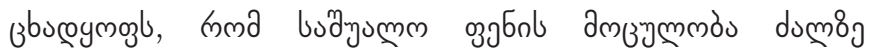

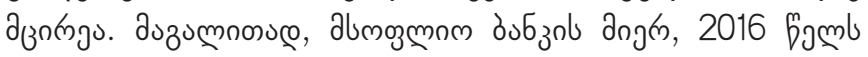

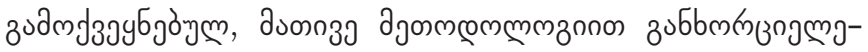

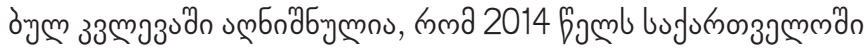

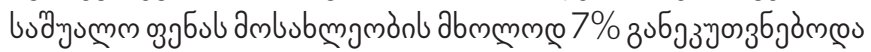

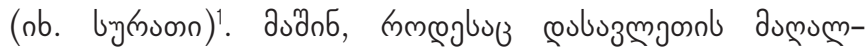

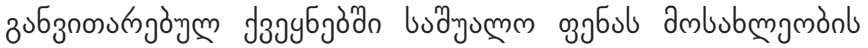

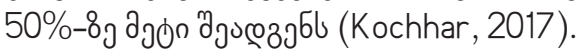

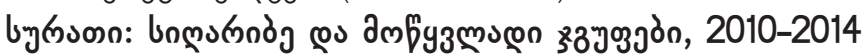

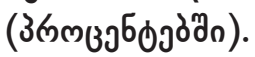

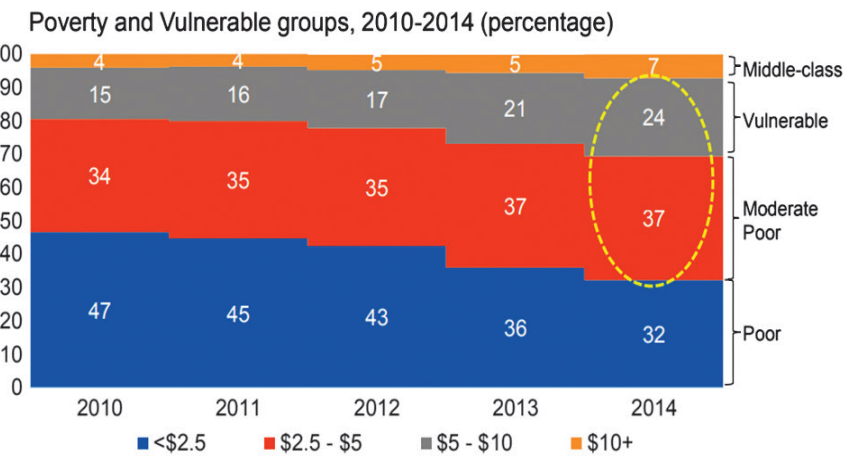

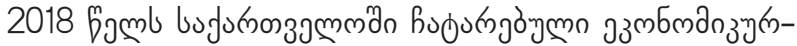

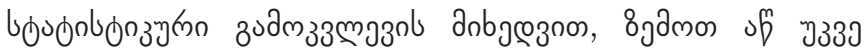

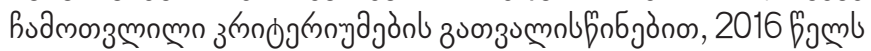

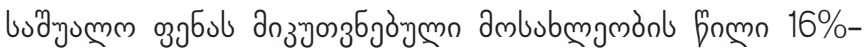

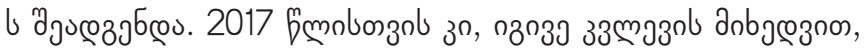

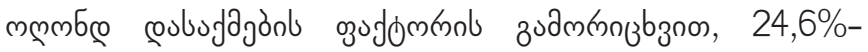

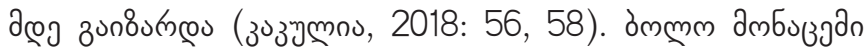

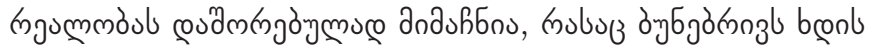

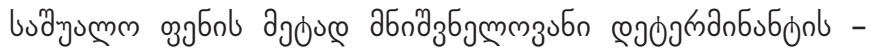

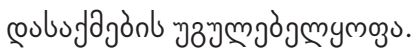

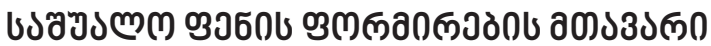

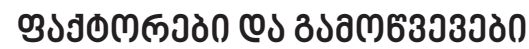

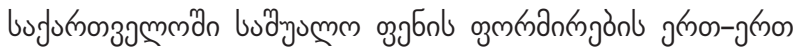

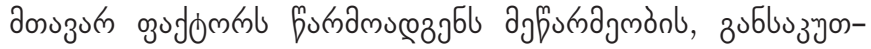

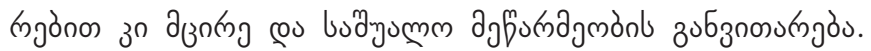

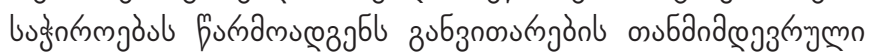

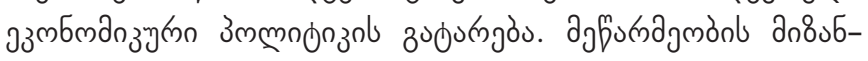

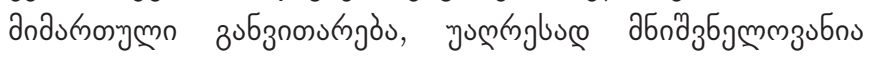

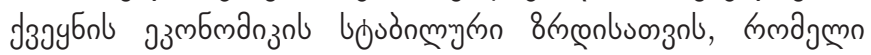

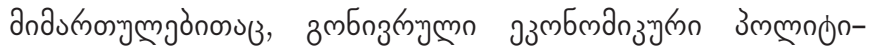

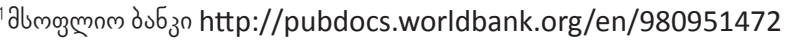
223098077/Georgia-PPA-FY16-presentation-AUG2016-final.pdf

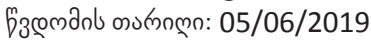




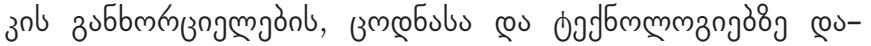

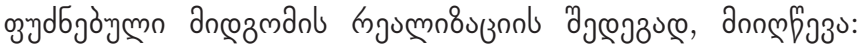

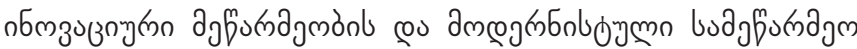

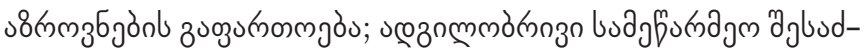

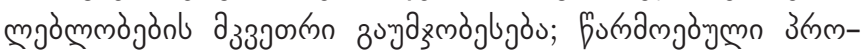

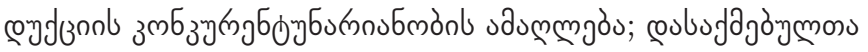

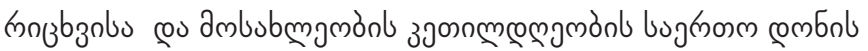
8 mons.

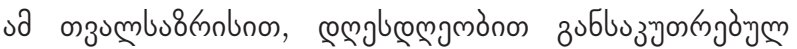

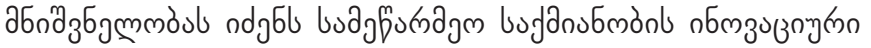

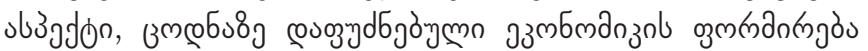

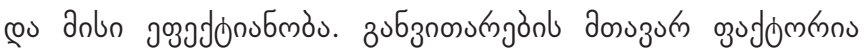

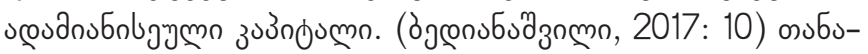

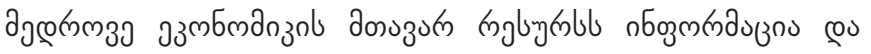

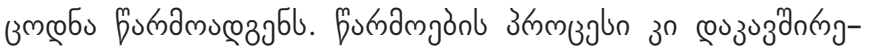

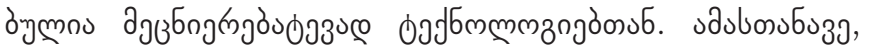

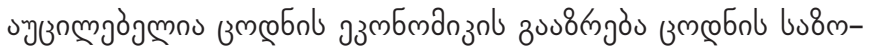

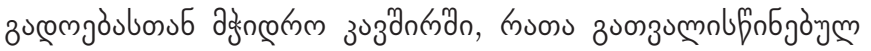

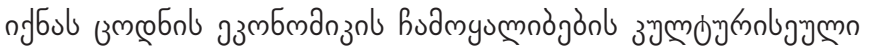

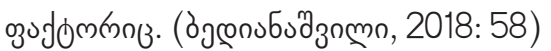

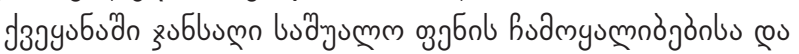

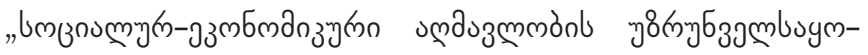

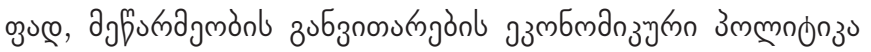

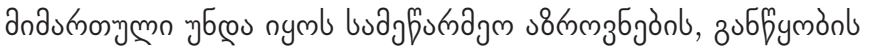

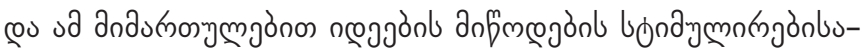

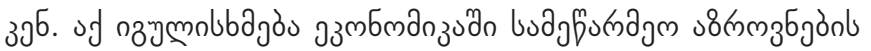

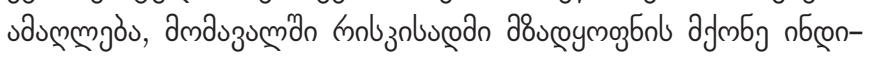

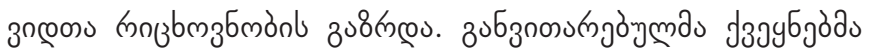

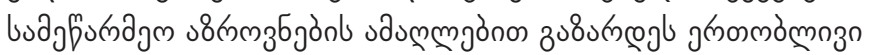

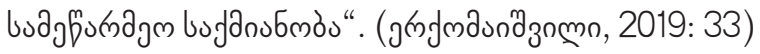

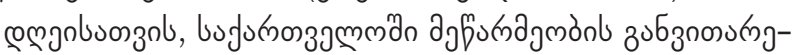
on ogaz з з

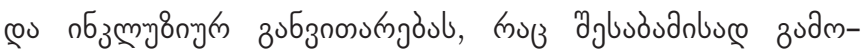

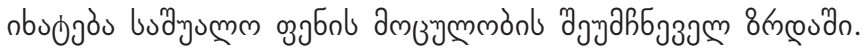

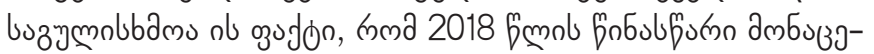

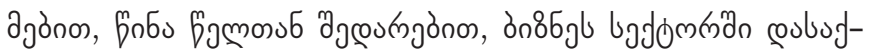

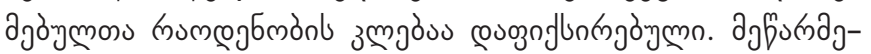

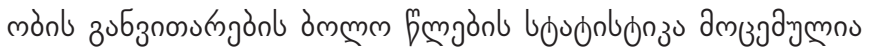

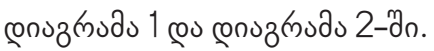

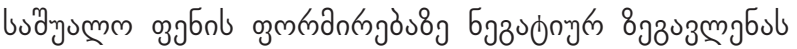

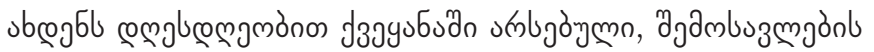

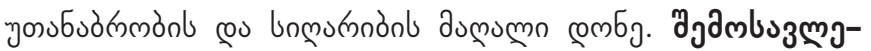
bol yosubs

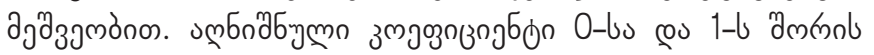

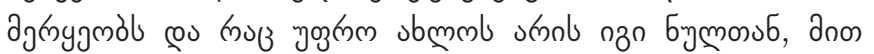
चoुरूल gmonl з з б

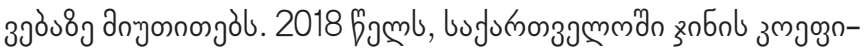

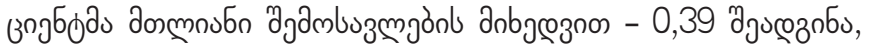

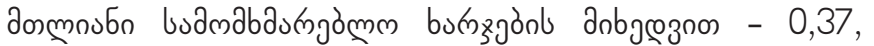

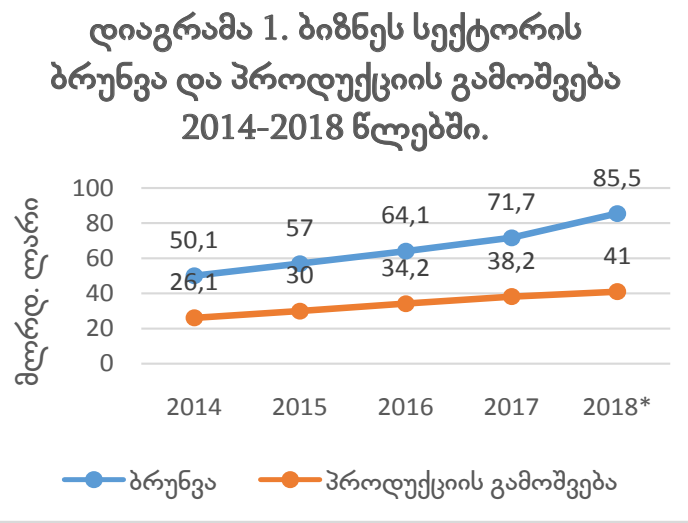

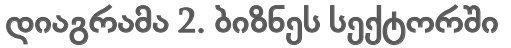

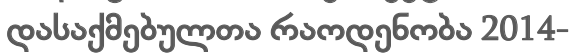

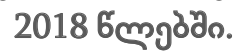

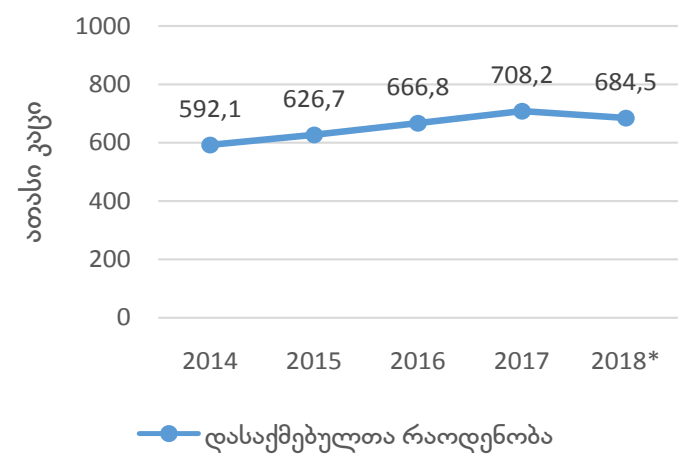

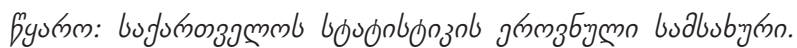

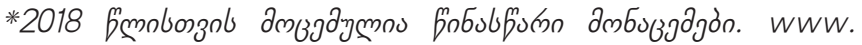
geostat.ge/ka/modules/categories/326/sacarmota-statistikuri-

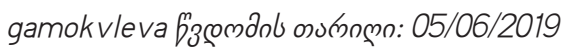

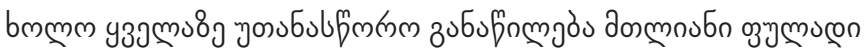

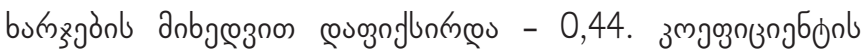

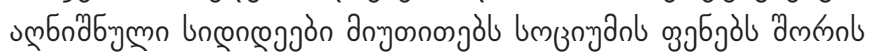

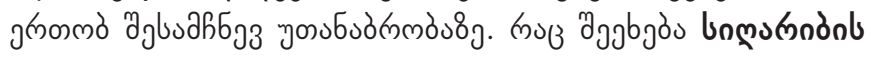

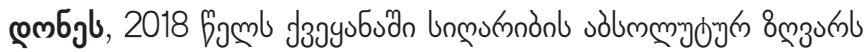

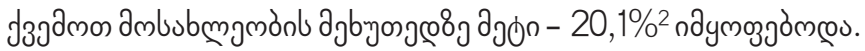

\section{esu336s}

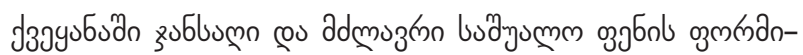

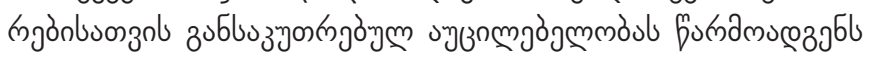

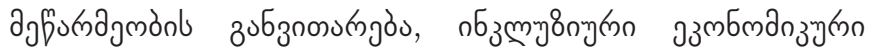

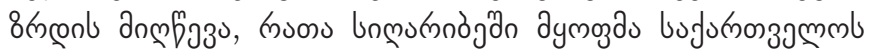

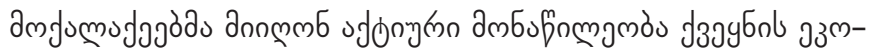

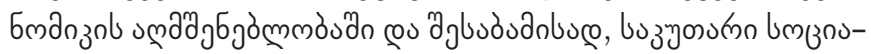

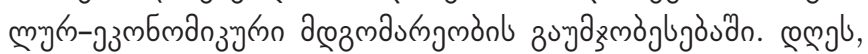

\footnotetext{
${ }^{2}$ fysłrm: www.geostat.ge/ka/modules/categories/192/tskhovrebis-

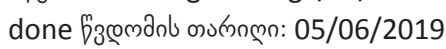




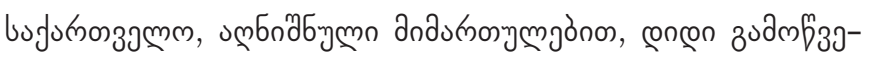

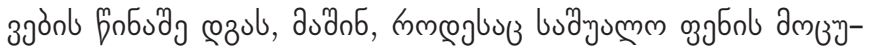

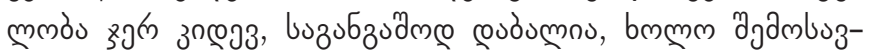

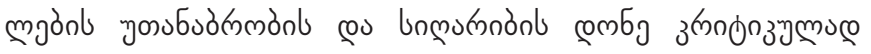
dugumonn.

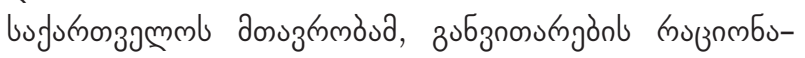

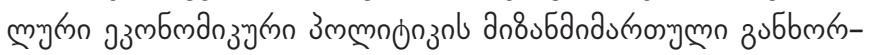

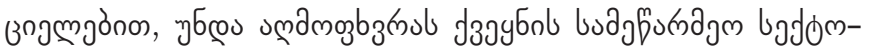

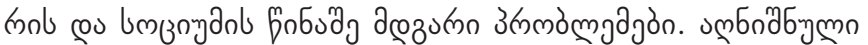
an85obuonzol bughnmms:

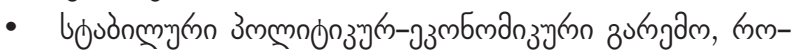

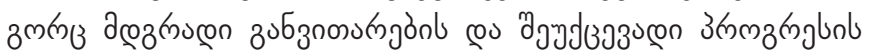
zomsobon;

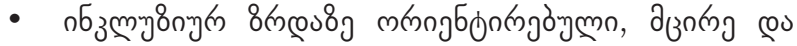

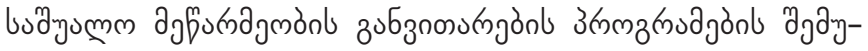

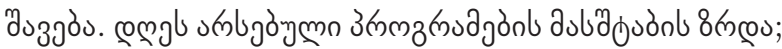

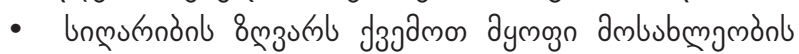

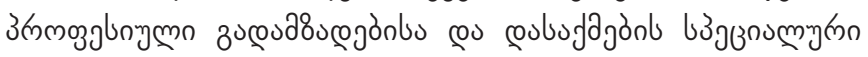

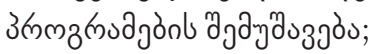

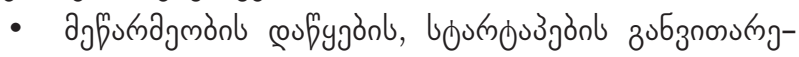

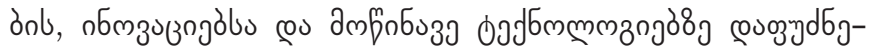

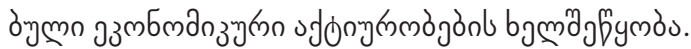

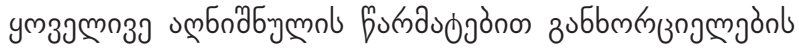

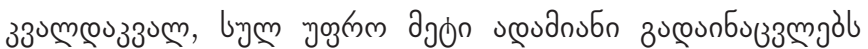

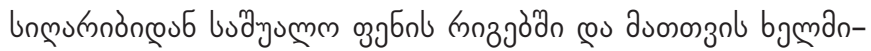

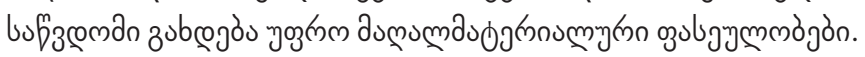

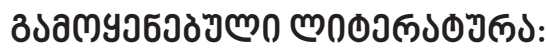

1. Bedianashvili, G. (2018) Culture as a Factor of Knowledge Economics with Paradigmatic Changes in Systemic Institutional Context [Kulturarogorts tsodnis ekonomikis formirebis faqtori paradigmuli tsvlilebebis sistemurinstituciuri konteqstit]. Journal "Globalization and Business", N6, P.58-66. (In Georgian).

2. Bedianashvili, G. (2017) Formation of Knowledge Economy and Innovative Entrepreneurial Policy: Institutional Aspects [Tsodnis ekonomikis formireba da inovaciurisamewarmeo politika: institutsiuri aspeqti]. Journal "Globalization and Business", N3, P.10-16 (In Georgian).

3. Erkomaishvili, G. (2019) Rational Economic Policy_Driving force from business to social entrepreneurship [Ratsionaluri ekonomikuri politika - biznesmetsarmeobidan socialuri metsarmeobisaken mamodzravebeli dzala]. Publishing House "UNIVERSALI", Tbilisi (In Georgian).

4. Kakulia, M., Kapanadze N. (2018) Middle Class in Georgia: Quantitative Evaluation, Dynamics and Profile [Sashualo klasi saqartveloshi: raodenobrivi shefaseba, dinamika da profile]. Friedrich Ebert Foundation, Tbilisi (In Georgian).

5. National Statistics Office of Georgia. (2018) Entrepreneurship in Georgia - Statistical Publication [Saqartvelos. Mewarmeoba Saqartveloshi - Statistikuri publikatsia]. Statistikis erovnuli samsakhuri. Tbilisi (In Georgian).

6. Chitanava, N. (2018) Challenges and Strategy of the Economy of Georgia [Saqartvelos ekonomikis gamotsvevebi da strategia]. Publishing House "IVERIONI", Tbilisi (In Georgian).

7. Kochhar, Rakesh (2017) Middle Class Fortunes in Western Europe. Luxembourg Income Study (LIS), asbl. https:// www.econstor.eu/bitstream/10419/169262/1/702.pdf

8. World Bank Group, GEORGIA: RECENT TRENDS AND DRIVERS OF POVERTY REDUCTION. August 17 2016. http:// pubdocs.worldbank.org/en/980951472223098077/Georgia-PPA-FY16-presentation-AUG2016-final.pdf

9. www.geostat.ge 


\title{
CHALLENGES OF MIDDLE CLASS FORMATION IN THE CONTEXT OF ENTREPRENEURSHIP DEVELOPMENT IN GEORGIA
}

\author{
AMIRANI MAGLAKELIDZE \\ PhD Student \\ Ivane Javakhishvili Tbilisi State University, Georgia \\ maglakelidze.amirani@gmail.com
}

KEYWORDS: MIDDLE CLASS; DEVELOPMENT OF ENTREPRENEURSHIP; INCOME INEQUALITY; POVERTY.

For citation: Maglakelidze, A. (2019). Challenges of Middle Class Formation in the Context of Entrepreneurship Development in Georgia, Globalization And Business, №8, pp. 116-120. https://doi.org/10.35945/gb.2019.08.014

\section{SUMMARY}

In order to ensure irreversible development and stable social-economic conditions of a State, it should have a strong middle class, defining the country's social and economic development targets. Considering the said, economic policy of development should be directed to the implementation of the measures supporting the formation of the healthy middle class in Georgia.

Share of the population belonging to the middle class is still low in Georgia. According to the studies conducted by the World Bank, in 2014 only 7\% of the population of Georgia belonged to the middle class. According to the economicstatistical study conducted in Georgia in 2018, share of the population belonging to the middle class in 2016 was $16 \%$. The same study, with exclusion of the employment factor, shows the growth of the said share to $24,6 \%$. Ignoring the employment - very important determinant of the middle class - naturally puts the last figure far from reality. Current income inequality and high level of poverty negatively impact formation of the middle class in the country.

In order to form a middle class, Government of Georgia must overcome the problems faced by country's entrepreneurship and society by means of the purposeful implementation of the rational policy of economic development. Achieving the said target requires: a stable political-economic environment; elaboration of the small, medium business development programs oriented on the inclusive growth, increase the scales of the currently existing program; development of the special professional training and employment programs for the population below the poverty line; promotion of economic activities based on the start of entrepreneurship, development of the start-ups, innovations and advanced technologies. 